\title{
Epigeic beetles (Coleoptera) of the Lake Świdwie nature reserve
}

\author{
ŁUKASZ BARAN, ${ }^{1}$ BRYGIDA RADAWIEC, ${ }^{2}$ ANDRZEJ ZAWAL, ${ }^{3}$ \\ ANNA NOWAK, ${ }^{4}$ PRZEMYSŁAW ŚMIETANA ${ }^{5}$
}

${ }^{1}$ University of Szczecin, Department of Invertebrate Zoology and Limnology, Institute for Research on Biodiversity, Faculty of Biology, Wąska 13, 71-415 Szczecin, Poland, e-mail: ukaszbaran84@gmail.com

${ }^{2}$ Institute of Biology and Environment protection of Pomeranian University, Arciszewskiego 22b, 76-200 Słupsk, Poland, e-mail: brygida.radawiec@apsl.edu.pl

${ }^{3}$ University of Szczecin, Department of Invertebrate Zoology and Limnology, Institute for Research on Biodiversity, Faculty of Biology, Wąska 13, 71-415 Szczecin, Poland, e-mail: andrzej.zawal@usz.edu.pl, ORCID ID: 0000-0002-5838-6060

${ }^{4}$ University of Szczecin, Department of Plant Taxonomy and Phytogeography, Institute for Research on Biodiversity, Faculty of Biology, Wąska 13, 71-415 Szczecin, Poland, e-mail: anna.nowak@usz.edu.pl

${ }^{5}$ University of Szczecin, Deparment of Ecology \& Environmental Protection, Institute for Research on Biodiversity, Faculty of Biology, 71-415 Szczecin, Wąska 13, Poland, e-mail: przemyslaw.smietana@usz.edu.pl

Keywords Coleoptera, fauna, species composition, Świdwie nature reserve

Abstract The data presented concern preliminary results of faunistic research carried out on the epigeic beetle fauna in the Lake Świdwie nature reserve, NW Poland (UTM VV53). Fourteen pitfall traps were placed in four different habitats in the reserve. A total of 957 specimens were collected belonging to 83 species and 16 families: Byrrhidae, Carabidae, Catopidae, Curculionidae, Dermestidae, Dryopidae, Elateridae, Eucinetidae, Hydrophilidae, Geotrupidae, Leiodidae, Limnichidae, Silphidae, Staphylinidae, Scarabaeidae, Tenebrionidae. Representatives of Staphylinidae, Hydrophilidae and Curculionidae were determinate to the family level only.

\section{Chrząszcze epigeiczne (Coleoptera) rezerwatu przyrody "Jezioro Świdwie"}

Słowa kluczowe Coleoptera, chrząszcze, fauna, skład gatunkowy, rezerwat przyrody „Jezioro Świdwie”

Streszczenie Przedstawiono wstępne wyniki badań faunistycznych chrząszczy epigeicznych w rezerwacie przyrody „Jezioro Świdwie” w Polsce północno-zachodniej (UTM VV53). Czternaście pułapek umieszczono w czterech różnych siedliskach. Odłowiono łącznie 957 okazów należących do 83 gatunków i 16 rodzin: Byrrhidae, Carabidae, Catopidae, Curculionidae, Dermestidae, Dryopidae, Elateridae, Eucinetidae, Hydrophilidae, Geotrupidae, Leiodidae, Limnichidae, Silphidae, Staphylinidae, Scarabaeidae, Tenebrionidae. Przedstawiciele Staphylinidae, Hydrophilidae i Curculionidae oznaczone tylko do poziomu rodziny. 


\section{Introduction}

The protected areas of Western Pomerania are relatively well known, but only for selected families of beetles. A study by Wolender (2013) summarizes the results of many years of research on one of the largest families of epigeic beetles, the Carabidae. This paper, however, presents material from forest, grassland and dune habitats. The little information available on carabids of marshland is presented in a faunistic study of the fauna of the island of Wolin (Radawiec et al., 2015).

The best known are the carabids of wetlands (rushes and sedges) of the buffer zone of the Leon Wyczółkowski Cisy Staropolskie reserve (Stachowiak, Wilcz, 2001).

Other families of epigeic beetles in the wetlands of Western Pomerania have not been the subject of special studies. There has been only preliminary research on the fauna of the epigeic beetles of the Lake Szare nature reserve (Aleksandrowicz, Dąbkowski, 2007) and a study on the fauna of Gmina Tuczno, where Gutowski and Ruta (2004) recorded 38 and 49 beetle species in peat bogs and wet grassland. However, Gutowski and Ruta (2004) did not use pitfall traps.

\section{Study area}

Lake Świdwie is located in West Pomerania, about $20 \mathrm{~km}$ northwest of Szczecin, in the Puszcza Wkrzańska forest (E14²1'41' N5333'50'). This shallow lake, together with the surrounding wetlands, is an ecologically valuable area, constituting one of the most important areas in the country on the bird migration path and under protection as the Świdwie nature reserve, which was added to the list of wetlands of international importance in 1978 under the Ramsar Convention.

At present, the reserve covers 891.28 ha, and the lake together with complexes of rushes covers a total area of 358 ha (Pienkowski, Kupiec, 2001). The surface area of the open water of the lake constitutes only $5.56 \%$ of its original area. The reserve area is fed by the Upper Gunica River and has a well-developed hydrographic network in the form of numerous natural watercourses and artificial canals (Kowalski, Bacieczko, 1993). The dominant communities having the most important role in the terrestrialization of the area are Phragmitetum australis, Typhetum angustifolie, Typhetum latifolie and Sparganietum erecti (Bacieczko, Kowalski, 1993). Dominant among grassland vegetation are Arrhenatheretum elatioris, Deschampsietosum caespitosae, Potentillo Festucetum arundinaceae, Calamagrostietum epigeji, Urtico Calystegietum sepium, Caricetum gracilis and Caricetum ripariae (Bacieczko, Kowalski, 1993). The forests in the reserve belong to the classes Alnetea glutinosae and Vaccinio piceetea (Kowalski, Bacieczko, 1993).

The study area was divided into 5 sites. The site numbers correspond to the locations marked in Figure 1. 


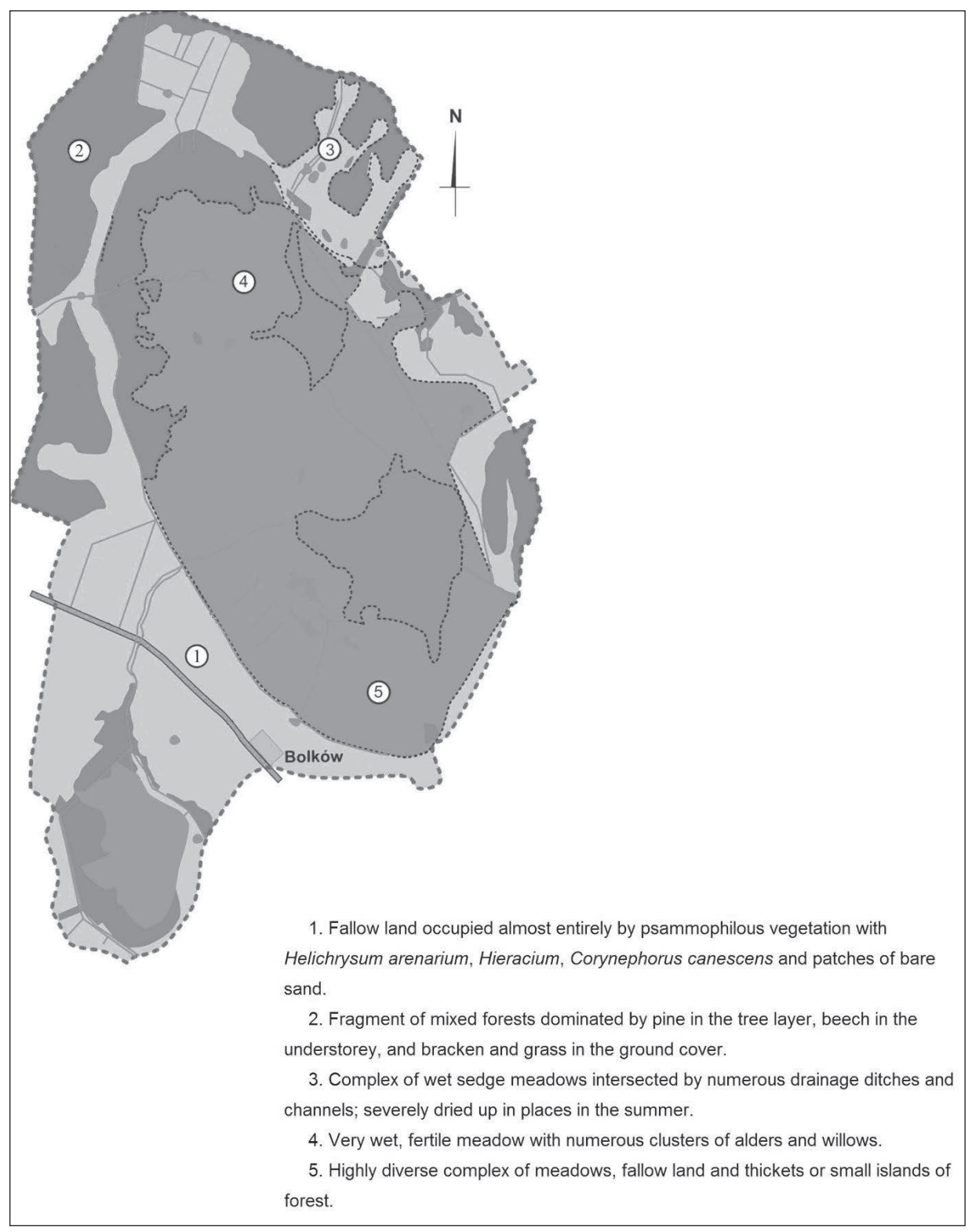

Figure 1. Map showing the distribution of the study sites in the Świdwie reserve 


\section{Research methods}

Three research cycles were carried out in 2010 in the Świdwie nature reserve on 15-21 June, 10-15 July and 17-22 August.

Epigeic beetles were caught using $500 \mathrm{ml}$ plastic cups. The upper rim of the cup, which was also the entrance to the trap, was $10 \mathrm{~cm}$ in diameter. The trap was placed in the ground so that the rim was level with the surface, to ensure that beetles penetrating the soil surface would fall in easily. To kill and preserve the insects entering the traps, they were filled with $50 \mathrm{ml}$ of ethylene glycol (25\%). The traps were arranged in a line, about 10 metres apart. Ten traps per habitat were set up in the line.

This is a standard, commonly used method in this type of research (Thiele, 1977).

The beetles were preserved in $70 \%$ ethyl alcohol and identified in the laboratory.

Works by Koch $(1989,1991)$ were used to determine species habitat preferences.

Similarity of species composition was evaluated using the Jaccard index and PAST software (Hammer et al., 2013).

\section{Results and conclusions}

During the study season a total of 957 epigeic beetles were caught, belonging to 16 families and 83 species (Table 1). Representatives of the families Staphylinidae, Hydrophilidae and Curculionidae were not identified to species.

There were 51 Carabidae species, 7 species each assigned to the Elateridae and Silphidae families, 3 each to Tenebrionidae and Leiodidae, 2 species each to Catopidae, Dryopidae, Scarabaeidae and Geotrupidae, and one each to Byrrhidae, Dermestidae, Eucinetidae and Limnichidae (Table 1).

Table 1. Species composition and number of specimens of selected epigeic beetle families caught in the Świdwie reserve

\begin{tabular}{|c|c|c|c|c|c|c|}
\hline Familia and species & 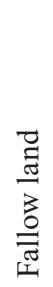 & 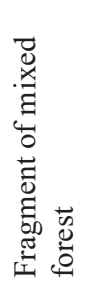 & 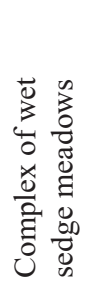 & 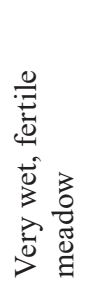 & 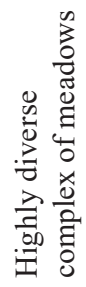 & Total \\
\hline 1 & 2 & 3 & 4 & 5 & 6 & 7 \\
\hline \multicolumn{7}{|l|}{ 1. Byrrhidae (Latreille, 1806) } \\
\hline Cytilus sericeus (Forster, 1771) & 1 & & & & & 1 \\
\hline \multicolumn{7}{|l|}{ 2. Carabidae (Latreille, 1802) } \\
\hline Abax parallelepipedus (Piller et Mitterpacher, 1783) & & 4 & & & & 4 \\
\hline Agonum emarginatum (Duftschmid, 1812) & & & & 7 & & 7 \\
\hline Agonum fuliginosum (Panzer, 1809) & & & 12 & 46 & 6 & 64 \\
\hline Agonum viduum (Panzer, 1796) & & & & 1 & & 1 \\
\hline Amara aenea (Degeer, 1774) & 1 & & & & & 1 \\
\hline Amara bifrons (Gyllenhal, 1810) & 2 & & & & & 2 \\
\hline
\end{tabular}




\begin{tabular}{|c|c|c|c|c|c|c|}
\hline 1 & 2 & 3 & 4 & 5 & 6 & 7 \\
\hline Amara familiaris (Duftschmid, 1812) & & & & & 1 & 1 \\
\hline Amara littorea (Thomson, 1857) & 1 & & & & & 1 \\
\hline Amara lunicollis (Schiødte, 1837) & & 2 & & 1 & 4 & 7 \\
\hline Amara spreta (Dejean, 1831) & 1 & & & & & 1 \\
\hline Badister sodalis (Duftschmid, 1812) & & & & 1 & & 1 \\
\hline Bembidion assimile (Gyllenhal, 1810) & & & 2 & 3 & & 5 \\
\hline Bembidion gilvipes (Sturm, 1825) & & & & 12 & & 12 \\
\hline Bembidion guttula (Fabricius, 1792) & & & & 1 & 1 & 2 \\
\hline Bembidion mannerheimii (C. Sahlberg, 1827) & & & 8 & 11 & 6 & 25 \\
\hline Calathus erratus (Sahlberg, 1827) & 2 & & & & & 2 \\
\hline Calathus fuscipes (Goeze, 1777) & 7 & 1 & 1 & & & 9 \\
\hline Calathus melanocephalus (Linnaeus, 1758) & 3 & & & & & 3 \\
\hline Carabus arcensis (Herbst, 1784) & & 1 & & & & 1 \\
\hline Carabus granulatus (Linnaeus, 1758) & & & 5 & 7 & 2 & 14 \\
\hline Carabus violaceus (Linnaeus, 1758) & & 4 & & & & 4 \\
\hline Clivina fossor (Linnaeus, 1758) & & & & 3 & 1 & 4 \\
\hline Dyschirius globosus (Herbst, 1784) & & & 8 & 4 & 2 & 14 \\
\hline Elaphrus uliginosus (Fabricius, 1792) & & & & & 1 & 1 \\
\hline Harpalus latus (Linnaeus, 1758) & 1 & 1 & 8 & & 2 & 12 \\
\hline Harpalus luteicornis (Duftschmid, 1812) & & & 1 & & 1 & 2 \\
\hline Harpalus pumilus (Sturm, 1818) & 1 & & & & & 1 \\
\hline Harpalus rufipes (Degeer, 1774) & 1 & & 1 & & & 2 \\
\hline Harpalus smaragdinus (Duftschmid, 1812) & 3 & & & & & 3 \\
\hline Harpalus tardus (Panzer, 1797) & 2 & & 2 & & & 4 \\
\hline Leistus ferrugineus (Linnaeus, 1758) & & 1 & & & & 1 \\
\hline Microlestes minutulus (Goeze, 1777) & 7 & 1 & 1 & & & 9 \\
\hline Olisthopus rotundatus (Paykull, 1790) & & & 1 & & & 1 \\
\hline Oodes helopioides (Fabricius, 1792) - VU & & & & 23 & 1 & 24 \\
\hline Oxypselaphus obscurus (Herbst, 1784) & & & & 4 & 1 & 5 \\
\hline Panagaeus cruxmajor (Linnaeus, 1758) & & & & & 2 & 2 \\
\hline Poecilus lepidus (Leske, 1785) & & 1 & & & & 1 \\
\hline Poecilus versicolor (Sturm, 1824) & & & 8 & & 14 & 22 \\
\hline Pterostichus diligens (Sturm, 1824) & & & & 7 & 1 & 8 \\
\hline Pterostichus minor (Gyllenhal, 1827) & & & & 4 & & 4 \\
\hline Pterostichus niger (Schaller, 1783) & & 16 & 3 & & 3 & 22 \\
\hline Pterostichus nigrita (Paykull, 1790) & & & 7 & & & 7 \\
\hline Pterostichus oblongopunctatus (Fabricius, 1787) & & 8 & & & & 8 \\
\hline Pterostichus rhaeticus (Heer, 1838) & & & & 2 & & 2 \\
\hline Pterostichus strenuus (Panzer, 1797) & & & & 2 & & 2 \\
\hline Pterostichus vernalis (Panzer, 1796) & & & & 7 & 2 & 9 \\
\hline Stenolophus mixtus (Herbst, 1784) & & & & 2 & & 2 \\
\hline Stomis pumicatus (Panzer, 1796) & & & 1 & & & 1 \\
\hline Syntomus foveatus (Fourcroy, 1785) & 2 & & & & & 2 \\
\hline Trechus obtusus (Erichson, 1837) - LC & & & 2 & & & 2 \\
\hline Trechus quadristriatus (Schrank, 1781) & & & 1 & & & 1 \\
\hline
\end{tabular}




\begin{tabular}{|c|c|c|c|c|c|c|}
\hline 1 & 2 & 3 & 4 & 5 & 6 & 7 \\
\hline \multicolumn{7}{|l|}{ 3. Catopidae (Thomson, 1862) } \\
\hline Catops fuliginosus (Erichson, 1837) & 1 & 5 & 1 & & 1 & 8 \\
\hline Sciodrepoides watsoni (Spence, 1815) & 3 & 2 & & & & 5 \\
\hline 4. Curculionidae (Latreille, 1802) & 19 & 16 & 8 & 5 & 7 & 55 \\
\hline 5. Dermestidae (Latreille, 1807) & 4 & 4 & 2 & & & 10 \\
\hline Dermestes laniarius (Illiger, 1801) & 4 & 4 & 2 & & & 10 \\
\hline \multicolumn{7}{|l|}{ 6. Dryopidae (Fleming, 1821) } \\
\hline Dryops ernesti (Des Gozis, 1886) & & & & 2 & 2 & 4 \\
\hline Dryops nitidulus (Heer, 1841) & & & & & 2 & 2 \\
\hline \multicolumn{7}{|l|}{ 7. Elateridae (Leach, 1815) } \\
\hline Agriotes lineatus (Linnaeus, 1767) & & & & & 2 & 2 \\
\hline Agriotes obscurus (Linnaeus, 1758) & & & & 4 & 6 & 10 \\
\hline Agrypnus murinus (Linnaeus, 1758) & 2 & 1 & & & 12 & 15 \\
\hline Hemicrepidius niger (Linnaeus, 1758) & & & & & 3 & 3 \\
\hline Hypnoidus riparius (Fabricius, 1792) & & & & 28 & & 28 \\
\hline Prosternon tesselatum (Linnaeus, 1758) & & 2 & & & & 2 \\
\hline Selatosomus aeneus (Linnaeus, 1758) & & 4 & & & & 4 \\
\hline \multicolumn{7}{|l|}{ 8. Eucinetidae (Lacordaire, 1857) } \\
\hline Eucinetus haemorrhoidalis (Germar, 1818) & 1 & & & & & 1 \\
\hline \multicolumn{7}{|l|}{ 9. Geotrupidae (Latreille, 1806) } \\
\hline Anoplotrupes stercorosus (Hartmann in L.G.Scriba, 1791) & & 2 & 1 & & & 3 \\
\hline Trypocopris vernalis (Linnaeus, 1758) & 3 & 22 & 7 & & & 32 \\
\hline \multicolumn{7}{|l|}{ 10. Limnichidae (Erichson, 1846) } \\
\hline Limnichus sericeus (Duftschmid, 1825) & & & & 8 & 6 & 14 \\
\hline \multicolumn{7}{|l|}{ 11. Scarabaeidae (Latreille, 1802) } \\
\hline Aphodius coenosus (Panzer, 1798) & 1 & & & & & 1 \\
\hline Phyllopertha horticola (Linnaeus, 1758) & & 3 & & & & 3 \\
\hline \multicolumn{7}{|l|}{ 12. Silphidae (Latreille, 1807) } \\
\hline Nicrophorus investigator (Zetterstedt, 1824) & 1 & 1 & & & & 2 \\
\hline Nicrophorus vespillo (Linnaeus, 1758) & 10 & & 1 & 2 & 5 & 18 \\
\hline Nicrophorus vespilloides (Herbst, 1783) & 1 & 10 & & & & 11 \\
\hline Phosphuga atrata (Linnaeus, 1758) & & 5 & & & & 5 \\
\hline Silpha carinata (Herbst, 1783) & & 54 & 12 & & & 66 \\
\hline Silpha tristis (Illiger, 1798) & & & & & 1 & 1 \\
\hline Thanatophilus sinuatus (Fabricius, 1775) & & & & & 1 & 1 \\
\hline 13. Staphylinidae (Latreille, 1802) & 22 & 16 & 113 & 67 & 68 & 286 \\
\hline \multicolumn{7}{|l|}{ 14. Tenebrionidae (Latreille, 1802) } \\
\hline Crypticus quisquilis (Linnaeus, 1761) & 5 & & & & & 5 \\
\hline Opatrum sabulosum (Linnaeus, 1761) & 2 & & & & & 2 \\
\hline Lagria hirta (Linnaeus, 1758) & & 2 & & & & 2 \\
\hline Total specimens & 110 & 189 & 217 & 264 & 167 & 957 \\
\hline Total spesies & 29 & 27 & 26 & 27 & 31 & 83 \\
\hline
\end{tabular}


Species richness was relatively even: the most species (31) were found in the highly diverse complex of meadows and the fewest (26) in the complex of wet sedge meadows. The number of individuals caught varied within a fairly large range: from 110 individuals in the fallow land with psammophilous vegetation to 264 individuals in the very wet, fertile meadow near Lake Świdwie Małe (Tabble 1).

No species common to all habitats were identified. The species composition of the families was highly varied (Figure 2). The highest similarity (about 36\%) was noted for the assemblages of wet meadows. These assemblages are very different from the others - diverse meadow, mixed forest and psammophilous vegetation, with a similarity index of only about $10 \%$.

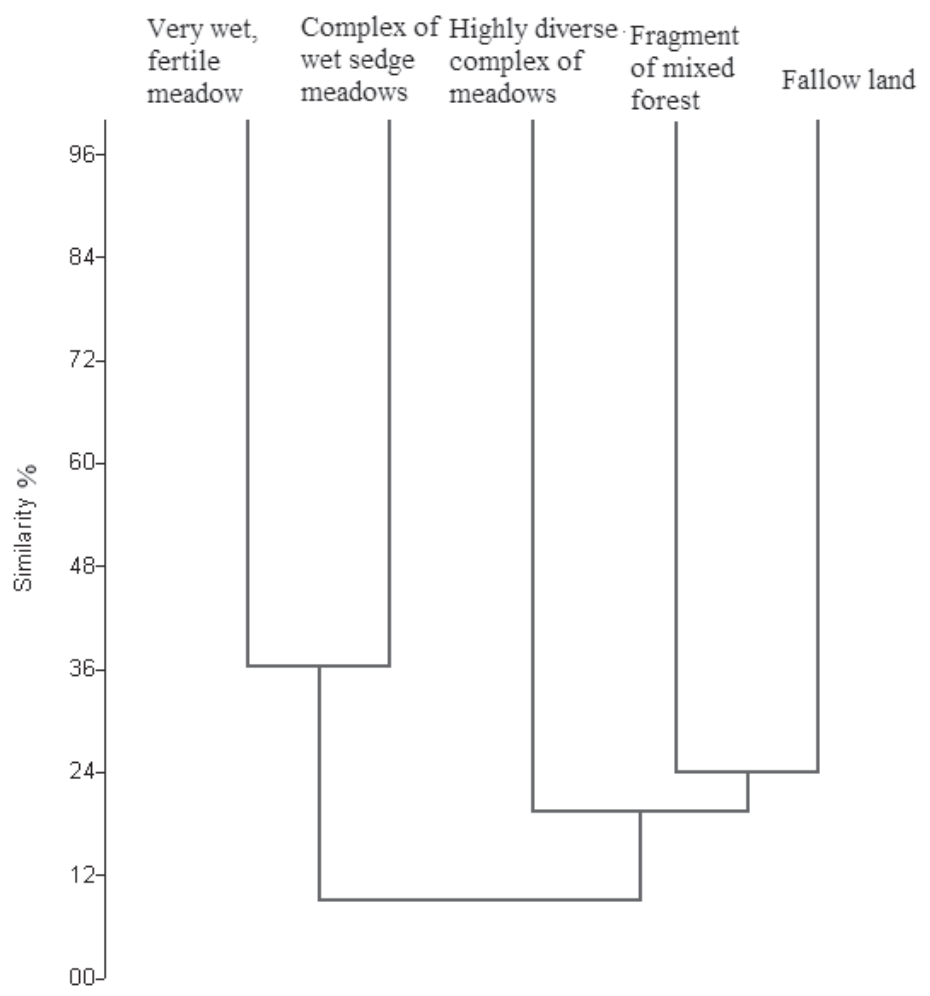

Figure 2. Similarity of the species composition of the habitats based on Jaccard's index

This indicates a great diversity of species composition and its evident dependence on the high moisture level at the site. Assemblages typical of wet habitats are on the left side of the dendrogram and assemblages of dry habitats are on the right (Figure 2).

Widely represented and fairly abundant stenobiontic hygrophilous species were noted in wet meadows: Agonum emarginatum, A. fuliginosum, Bembidion gilvipes, B. guttula, B. mannerheimii, Carabus granulatus, Oodes helopioides, Oxypselaphus obscurus, Panagaeus cruxmajor, Pterostichus rhaeticus, P. vernalis, Stenolophus mixtus, Dryops ernesti, D. nitidulus, Hypnoidus riparius and Limnichus sericeus. 
Mesophilic grassland species are fairly abundant on the meadow composed of diverse habitats: Poecilus versicolor, Dyschirius globosus and Harpalus latus.

Mesophilic, stenobiontic forest species are dominant in the mixed forest: Carabus violaceus, Pterostichus niger, P. oblongopunctatus, Abax parallelepipedus, Silpha carinata, Phosphuga atrata, Nicrophorus vespilloides and Trypocopris vernalis.

Mesoxerophilic and xerophilic open-area species were noted in the psammophilous vegetation: Amara aenea, Amara bifrons, Amara littorea, Amara spreta, Calathus erratus, Harpalus pumilus, H. smaragdinus, H. tardus, Microlestes minutulus, Syntomus foveatus, Crypticus quisquilis and Opatrum sabulosum.

Ecologically valuable species with varying conservation status included Oodes helopioides (VU), and Trechus obtusus (LC). Oodes helopioides was most abundant in the wet meadow by Lake Świdwie Małe. Trechus obtusus was represented by single specimens.

The research is preliminary; the list of epigeic beetle species will increase considerably when the seasonal study period has been extended and other research methods have been applied.

\section{References}

Barber, H.S. (1931). Traps for cave inhabiting insects. J. Elisha Mitchell Sci. Soc., 46, 259-266.

Błoszyk, J., Bajaczyk, R., Błoszyk, G., Napierała, A. (2002). Uropodina (Acari: Mesostigmata) parków narodowych Polski na tle innych obszarów. Kosmo, 4 (51), 463-470.

Brauns, A. (1975). Owady leśne. Warszawa: PWRiL.

Browarski, B. (2006). Zróżnicowanie ekologiczne fauny biegaczowatych (Coleoptera, Carabidae) kompleksu torfowiskowego „Uroczysko Torfiaki”.

Burakowski, B., Mroczkowski, M., Stefańska, J. (1983). Chrząszcze - Coleoptera Scarabaeoidea, Dascilloidea, Byrrhoidea i Parnoidea. Katalog Fauny Polski. Warszawa: PWN.

Dart R.C. (2004). Medical Toxicology. Lippincott Williams \& Wilkins.

Eberling, L., Eberling, M. (1984). Faunistiche und ökologische Untrsuchungen zur Sukzession aasbesuchender Coleopteran im südlichen Eggegebirge. Dechanian, 139, 231-240

Frank, L.H. (1988). Paederus, sensu lato (Coleoptera: Staphylinidae): An Index and Review of the Taxa. Insecta Mundi, 2 (2). Retrieved from: http://baza.biomap.pl/pl/taxon/species.

Karpiński, J.J., Makólski, J. (1954). Biegaczowate (Carabidae, Coleoptera) w biocenozie lasu Białowieskiego Parku Narodowego. Roczn. Nauk Leśnych, 5 (121), 105-136.

Koch, K. (1991). Die Käfer Miteleuropas. Ökologie. Band 2. Pselaphidae - Lucanidae. Goecke und Evers, Krefeld.

Koechler, W., Schinder, Z. (1972). Owady naszych lasów. Warszawa: PWRiL.

Kowalski, W., Bacieczko, W. (1993). Charakterystyka geobotaniczna rezerwatu faunistycznego „Jezioro Świdwie”. Zesz. Nauk. AR Szczec. Roln., 54 (155), 98-124.

Łabędzki, A. (1984). Przyczynek do znajomości fauny biegaczowatych (Coleoptera: Carabidae) drzewostanów sosnowych różnych klas wieku w Nadleśnictwie Doświadczalnym Zielonka. Roczn. Akademii Rolniczej w Poznaniu, 152, 27-33.

Łęgowski, D. (2000). Przykład wykorzystania pająków (Aranei) jako bioindykatorów zmian zachodzących w środowisku leśnym. Sylwan, 6 (144), 53-62.

Marczak, D. (2008) Zróżnicowanie zgrupowań chrząszczy epigeicznych w siedliskach boru sosnowego świeżego. 
Skłodowski, J.W. (1995). Wpływ skrzenia środowiska na faunę Carabidae borów sosnowych Polski. Sylvan, 2, 69-86.

Sławska, M. (2000). Możliwości wykorzystania fauny glebowej do oceny efektywności zabiegów gospodarczych. Sylvan, 3 (144), 93-100.

Stebnicka, Z. (1976). Żukowate - Scarabaeidae. Grupa podrodzin: Scarabaeidae laparostici. Klucze do oznaczania owadów Polski. Warszawa: PWN.

Thiele, H.-U. (1977). Carabid beetles in their environments. A study on habitat selection by adaptations in physiology and behaviour. Berlin-Heidelberg-New York.

Tischler, W.H. (1976). Untersuchungen über die tierische Besiedlung von Aas in verschiedenen Strata von Waldökosystemen. Pedobiologia, 16, 99-105.

Maciejewski, K.H. (1998). Ocena bioróżnorodności środowisk leśnych Puszczy Boreckiej na przykładzie chrzaszczy epigeicznych. Rozprawa doktorska, maszynopis. Toruń: Uniwersytet im. Mikołaja Kopernika.

Skłodowski, J., Byk, A., Malinowska, A., Spała, S., Błędowski, J. (1998). Występowanie przedstawicieli rodziny żuka (Geotrupes Latreille) na zrębie z pozostawionymi kępami sosen. Sylvan, 11 (142), 37 42.

Pukowski, E. (1933). Ökologische Untersuchungen and Nicrophorus. F. Z. Morph Ökol Tiere. 27, 518-596.

Olechowicz, E. (2003). Entomofauna gleby i runa różnych ekosystemów Puszczy Kampinoskiej. In: R. Andrzejewski (ed.), Kampinoski Park Narodowy, Tom I, Przyroda Kampinoskiego Parku Narodowego (pp. 511-523). Izabelin.

Pieńkowski, P., Kupiec, M. (2001). Proces zarastania Jeziora Świdwie i zmiany w użytkowaniu jego otoczenia od początku XIX do końca XX wieku. Politechnika Zielonogórska, Zeszyty Naukowe. Inżynieria Środowiska, 11 (125), 265-277.

Cite as: Baran, Ł., Radawiec, B., Zawal, A., Nowak, A., Śmietana, P. (2017). Epigeic beetles (Coleoptera) of the Lake Świdwie nature reserve. Acta Biologica, 24, 29-37. DOI: 10.18276/ab.2017.24-04. 